\title{
Fomitiporia punicata and Phaeoacremonium minimum associated with Esca complex of grapevine in China
}

Qingtong Ye $\mathrm{e}^{1,2}$, Jingyi Jia', Ishara S. Manawasinghe ${ }^{1}$, Xinghong $\mathrm{Li}^{1}$, Wei Zhang ${ }^{1}$, Laura Mugnai ${ }^{3}$, Xuehong $\mathrm{Wu}^{2}$, Kevin D. Hyde ${ }^{4}$ and Jiye Yan ${ }^{1 *}$

\begin{abstract}
The Esca disease complex includes some of the most important trunk diseases of grapevines (Vitis spp.) and causes serious yield losses in grape production worldwide. However, there has been no detailed study on its presence and associated pathogens in China. During 2017-2019, a preliminary field survey was conducted in eight vineyards in Hebei and Ningxia provinces, China when unusual foliar symptoms were observed. Symptoms were distinct tiger striped leaves, which are typical of grapevine leaf stripe disease (GLSD), one of the most common diseases in the Esca complex. Tiger-stripe leaf symptoms were found in four vineyards, and incidence was cultivar dependent varying with vineyard and year. A total of 266 fungal isolates were obtained from wood tissues of grapevines with typical foliar symptoms of GLSD. Based on morphological characters and multigene-combined phylogenetic analyses, the Ascomycete Phaeoacremonium minimum, one of the pathogens associated with Esca complex was identified. The basidiomycete Fomitiporia punicata, which has never been reported infecting grapevine, was also identified and found to be associated with wood rot in grapevine. The remaining isolates included some known wood pathogens, such as Neofusicoccum species and Diaporthe species. Koch's postulates were performed in the greenhouse, confirming that both F. punicata and P. minimum caused leaf interveinal chlorosis and necrosis that resembled the GLSD symptoms of the Esca complex observed in the field. The present study provides the first detailed report of the Esca complex in China. In addition, this is the first record of F. punicata associated with Esca complex of grapevine worldwide. The results of this study provide new insights into the knowledge of the Esca complex.
\end{abstract}

Keywords: Grapevine trunk diseases (GTDs), Pathogenicity, Vitis vinifera

\section{Background}

Grape is one of the most important fruit crops in the world, and also the most economically important for making wine, beverages and other products. In 2018, the global grape cultivation area was 7,449,000 ha, of which China accounted for $12 \%$ and ranked in second place (OIV 2019). Grapevine trunk diseases (GTDs)

\footnotetext{
*Correspondence: jiyeyan@vip.163.com

'Beijing Key Laboratory of Environment Friendly Management on Fruit Diseases and Pests in North China, Institute of Plant and Environment Protection, Beijing Academy of Agriculture and Forestry Sciences, Beijing 100097, China

Full list of author information is available at the end of the article
}

have been reported in recent years in some vineyards in China, especially in mature vineyards (Yan et al. 2013). The most important grapevine trunk disease in China is Botryosphaeria dieback, which was first reported by Li et al. (2010). The main symptoms associated with Botryosphaeria dieback are fruit dropping and wood cankers (Yan et al. 2013). Botryosphaeria dieback causes a $10-20 \%$ reduction in yield and eventual death of vines in extreme environmental conditions such as long periods of high temperature or rainy seasons ( $\mathrm{Li}$ et al. 2010). Six Botryosphaeriaceae species (Botryosphaeria dothidea, Diplodia seriata,

(c) The Author(s). 2021 Open Access This article is licensed under a Creative Commons Attribution 4.0 International License, which permits use, sharing, adaptation, distribution and reproduction in any medium or format, as long as you give

appropriate credit to the original author(s) and the source, provide a link to the Creative Commons licence, and indicate if changes were made. The images or other third party material in this article are included in the article's Creative Commons licence, unless indicated otherwise in a credit line to the material. If material is not included in the article's Creative Commons licence and your intended use is not permitted by statutory regulation or exceeds the permitted use, you will need to obtain permission directly from the copyright holder. To view a copy of this licence, visit http://creativecommons.org/licenses/by/4.0/ 
Lasiodiplodia theobromae, Lasiodiplodia pseudotheobromae, Neofusicoccum parvum and Neofusicoccum mangiferae) associated with Botryosphaeria dieback in China (Li et al. 2010; Yan et al. 2013; Dissanayake et al. 2015b, 2015c). Another emerging grapevine trunk disease in China is Diaporthe dieback (Dissanayake et al. 2015a), and ten Diaporthe species (Diaporthe eres, D. hongkongensis, D. phaseolorum, D. sojae, D. guangxiensis, D. hubeiensis, D. viniferae, D. gulyae, D. pescicola and D. unshiuensis) associated with the disease in China (Dissanayake et al. 2015a; Manawasinghe et al. 2019). Esca disease complex was first reported in China in 2020, and P. minimum was the pathogen associated with the symptoms ( $\mathrm{Ye}$ et al. 2020).

Esca complex, some of which show typical leaf symptoms, includes the most devastating diseases on grapevine causing serious economic losses in many grapegrowing countries and regions (Mugnai et al. 1999; Gramaje et al. 2015; Spies et al. 2018; Guerin-Dubrana et al. 2019). The average incidence of Esca increased to 32.6\% in mature vineyards of Marche region, central eastern Italy from 2005 to 2007 (Romanazzi et al. 2009). The Esca complex is characterized by five different diseases based on wood symptoms, foliar symptoms, life stages of the vines and the causal agents, i.e., dark wood streaking, Petri disease, grapevine leaf stripe disease (GLSD), white rot and Esca proper (Mugnai et al. 1999; Surico 2009; Gubler et al. 2015; Mondello et al. 2018). This disease complex exhibits both external (e.g., symptomatic leaves and berries) and internal (i.e., diseased wood and branches) symptoms (Mugnai et al. 1999; Surico 2009; Gubler et al. 2015). The most characteristic foliar symptoms of the Esca complex are seen in the leaves, which develop a tiger-stripe pattern (Mugnai et al. 1999; Surico 2009; Gubler et al. 2015), and small reddish or dark purple spots on berries, which are known as "black measles" (Mugnai et al. 1999). Another common symptom in vineyards is vine apoplexy (Mugnai et al. 1999). The trunk (scion and sometimes rootstock) and main branches show several types of wood discoloration and necrosis (Mugnai et al. 1999; Surico 2009; Gubler et al. 2015). The internal symptoms in wood vary with the age of symptomatic grapevines and the pathogens involved (Mugnai et al. 1999; Fischer 2006; Cloete 2015; Mondello et al. 2018).

Esca disease was recently reported in China (Ye et al. 2020), but no detailed study has been conducted on the causal organisms or their pathogenicity. The present research was based on surveys and samples collection from Hebei and Ningxia provinces in China. Hebei Province has a long history of grape cultivation (Chu and Chen 2018). Ningxia Province is regarded as one of the best ecological zones in China for grape cultivation, mainly attributed to its suitable climate, such as adequate rain and sunshine, and large temperature difference between day and night, especially the low temperatures and less rain in autumn, and now it has developed into the largest wine grape-growing region in northwest China (Chen 2018). The objectives of this study were to (i) conduct a preliminary vineyard survey to determine the extent of Esca complex in Hebei and Ningxia provinces in China; (ii) investigate wood symptoms associated with GLSD; (iii) identify fungal pathogens associated with GLSD based on morphological characters and multigene-combined phylogenetic analyses; and (iv) evaluate the pathogenicity of some of the fungi associated with Esca symptoms.

\section{Results}

Field surveys

A field survey was conducted in eight vineyards in Hebei and Ningxia provinces in China (Table 1). The presence of foliar symptoms was recorded during the growing season, and the internal wood symptoms of the symptomatic vines were observed in late summer (Fig. 1a-f). Tiger-stripe foliar symptoms, the typical symptoms of GLSD, were observed in four ( $\mathrm{Hb}-1, \mathrm{Nx}-1, \mathrm{Nx}-4$ and $\mathrm{Nx}-5$ ) of eight surveyed vineyards, while no obvious symptoms were found in the other four vineyards ( $\mathrm{Hb}-2$, $\mathrm{Hb}-3, \mathrm{Nx}-2$ and Nx-3) (Table 1). Among the 7670 plants examined, 95 plants showed tiger-stripe foliar symptoms, and no apoplectic symptom was found during the survey. The incidence of tiger stripe-like foliar symptoms was quite variable from year-to-year, and also varied a lot between cultivars, both of which are well-known characteristics of this disease. For example, the incidence of the tiger-stripe foliar symptoms at $\mathrm{Hb}-1$ was low in 2017 (1.0\%) but much higher in 2018 (5.6\%); the incidence of the disease between cultivars in the same year and vineyard were obviously different $(0.7 \%$ on Merlot, $5.0 \%$ on Cabernet Franc, $0.3 \%$ on Marselan, $16.7 \%$ on Petit Verdot in 2018). Internal necrosis and decay in wood were randomly analysed in some vines with typical foliar symptoms of GLSD in vineyards $\mathrm{Hb}-1, \mathrm{Nx}-1$ and $\mathrm{Nx}-4$, and the main wood symptoms detected in 12 vines are shown in Additional file 1: Table S1.

\section{Fungal isolation, phylogenetic analyses and taxonomy}

Fungi were isolated from discoloured grapevine wood tissues and initially grouped on the basis of their morphological characteristics. From different types of symptomatic wood tissues, 266 fungal isolates were obtained. The fungal species associated with the main internal wood symptoms were determined (Additional file 1: Table S2). In a 19-year-old grapevine showing wood rot in vineyard $\mathrm{Hb}-1$, the most abundant types of fungi according to morphological characters were Fomitiporia- 
Table 1 Incidence of Esca in vineyards in Hebei and Ningxia provinces from 2017 to 2019

\begin{tabular}{|c|c|c|c|c|c|c|c|}
\hline Region & Vineyard & $\begin{array}{l}\text { Age of } \\
\text { vineyard in } 2017\end{array}$ & Year & $\begin{array}{l}\text { No. of } \\
\text { standing } \\
\text { plants }\end{array}$ & $\begin{array}{l}\text { No. of } \\
\text { symptomatic } \\
\text { plants }\end{array}$ & Vine cultivar & $\begin{array}{l}\text { Average incidence (incidence of Esca on } \\
\text { different cultivars) }\end{array}$ \\
\hline \multirow[t]{6}{*}{$\begin{array}{l}\text { Hebei } \\
\text { Province }\end{array}$} & $\mathrm{Hb}-1$ & 18 & 2017 & 1200 & 12 & $\begin{array}{l}\text { Merlot, "Cabernet type", } \\
\text { Marselan, Petit Verdot }\end{array}$ & $\begin{array}{l}\text { 1.0\% (Merlot 0.0\%, Petit Verdot 0.0\%, } \\
\text { "Cabernet type" 3.0\%, Marselan 0.7\%) }\end{array}$ \\
\hline & & 19 & 2018 & 1200 & 68 & $\begin{array}{l}\text { Merlot, "Cabernet type", } \\
\text { Marselan, Petit Verdot }\end{array}$ & $\begin{array}{l}\text { 5.6\% (Merlot 0.7\%, "Cabernet type" 5.0\%, } \\
\text { Marselan 0.3\%, Petit Verdot 16.7\%) }\end{array}$ \\
\hline & $\mathrm{Hb}-2$ & 6 & 2017 & 510 & 0 & Unknown & $0 \%$ \\
\hline & & 7 & 2018 & 560 & 0 & Unknown & $0 \%$ \\
\hline & $\mathrm{Hb}-3$ & 18 & 2017 & 960 & 0 & $\begin{array}{l}\text { "Cabernet type", Petit } \\
\text { Manseng }\end{array}$ & $0 \%$ \\
\hline & & 19 & 2018 & 240 & 0 & Unknown & $0 \%$ \\
\hline \multirow[t]{5}{*}{$\begin{array}{l}\text { Ningxia } \\
\text { Province }\end{array}$} & $\mathrm{Nx}-1$ & 5 & 2018 & Unknown & Unknown & Marselan, Chardonnay & $\begin{array}{l}\text { Based on wine grower's comment: some } \\
\text { diseased plants were found in the field }\end{array}$ \\
\hline & $N x-2$ & Unknown & 2018 & 2000 & 0 & Unknown & $0 \%$ \\
\hline & $N x-3$ & Unknown & 2019 & 560 & 0 & Unknown & $0 \%$ \\
\hline & $N x-4$ & 10 & 2019 & 200 & 5 & Unknown & $4.0 \%$ \\
\hline & $N x-5$ & Unknown & 2019 & 240 & 10 & Merlot & $4.2 \%$ \\
\hline
\end{tabular}

like and Phaeoacremonium-like. The remaining isolates were grouped as Botryosphaeriaceae species and Diaporthaceae species. A high percentage of Fomitiporia species were found from rotten wood in vineyard $\mathrm{Hb}-1$. Phaeoacremonium isolates were obtained from black streaks, symptomatic pith tissues and asymptomatic wood tissues at the base of trunk and rootstock in two vines in vineyards $\mathrm{Nx}-1$ and $\mathrm{Hb}-1$. Botryosphaeriaceae species were isolated from wood tissues with symptoms of white rot, black streaks and brown necrosis in vineyards $\mathrm{Hb}-1$ and $\mathrm{Nx}-1$. Diaporthaceae species were isolated from brown necrotic tissues in vineyard $\mathrm{Hb}-1$. Most of these fungi were isolated from the same diseased vines, confirming that several different pathogens often occur in the same grapevine at the same time in the field, as is well known for GTDs.

Major characteristics of the two species, F. punicata and $P$. minimum, are as follows:

The morphological characteristics of Fomitiporia-like isolates (cottony to woolly vegetative mycelium, with yellowish to brownish aerial hyphae, clamp connections present in hyphae, no conidia) were identical on PDA, thus two isolates (JZB3350001 and JZB3350002) were selected for molecular analysis. To identify the isolates at the species level, phylogenetic analyses were conducted based on LSU, ITS and tef1 sequences. The combined dataset of Fomitiporia species consisted of two isolates (JZB3350001 and JZB3350002) obtained in this study and sequences of 78 taxa retrieved from GenBank (Groenewald et al. 2001; Chen and Cui 2017) (Table 2). Phellinus uncisetus MUCL 46231 and P. uncisetus MUCL 47061 were used as outgroup taxa. The dataset consisted of 2477 total characters of which 1575 were constant and 208 were parsimony-uninformative. A heuristic search of the remaining 609 parsimonyinformative characters produced 1000 most parsimonious trees with a length of 1796 steps $(\mathrm{CI}=0.547, \mathrm{RI}=$ $0.774, \mathrm{RCI}=0.423$ and $\mathrm{HI}=0.453$ ). Topology of the maximum likelihood tree was similar to the maximum parsimony tree. The best scoring RAxML tree with a final likelihood value of $-15,647.433442$ is presented in Fig. 2. In the phylogenetic analysis, JZB3350001 and JZB3350002 clustered together with $F$. punicata, with 94\% (ML) and 95\% (MP) bootstrap values, respectively.

Fomitiporia punicata Y.C. Dai, B.K. Cui \& Decock in Dai et al. (2008) (Fig. 3).

Pathogenic on trunk and rootstock of Vitis vinifera. Asexual morph: no spores were produced on PDA or malt extract agar (MEA) cultures incubated at $25^{\circ} \mathrm{C}$ in the dark for more than 3 months. Sexual morph: undetermined.

Culture characteristics: Colonies on PDA reached $38.8 \pm 7.5 \mathrm{~mm}$ diameter after 10 days of incubation at $25^{\circ} \mathrm{C}$, yellowish, with cottony to wooly vegetative mycelium, with yellowish to brownish aerial hyphae, clamp connections, no conidia (Fig. 3c, d).

Material examined: China, Hebei Province, Huailai County, on trunk and rootstock of $V$. vinifera, 10 August 2018, Qingtong Ye and Xinghong Li, living cultures JZB3350001 and JZB3350002.

To confirm the species of Phaeoacremonium isolated in this study, phylogenetic trees were constructed from combined actin and $\beta$-tubulin gene sequences. The concatenated data set of Phaeoacremonium species comprised 68 taxa (Groenewald et al. 2001; Spies et al. 2018) (Table 2), including eight isolates (JZB3190002, 


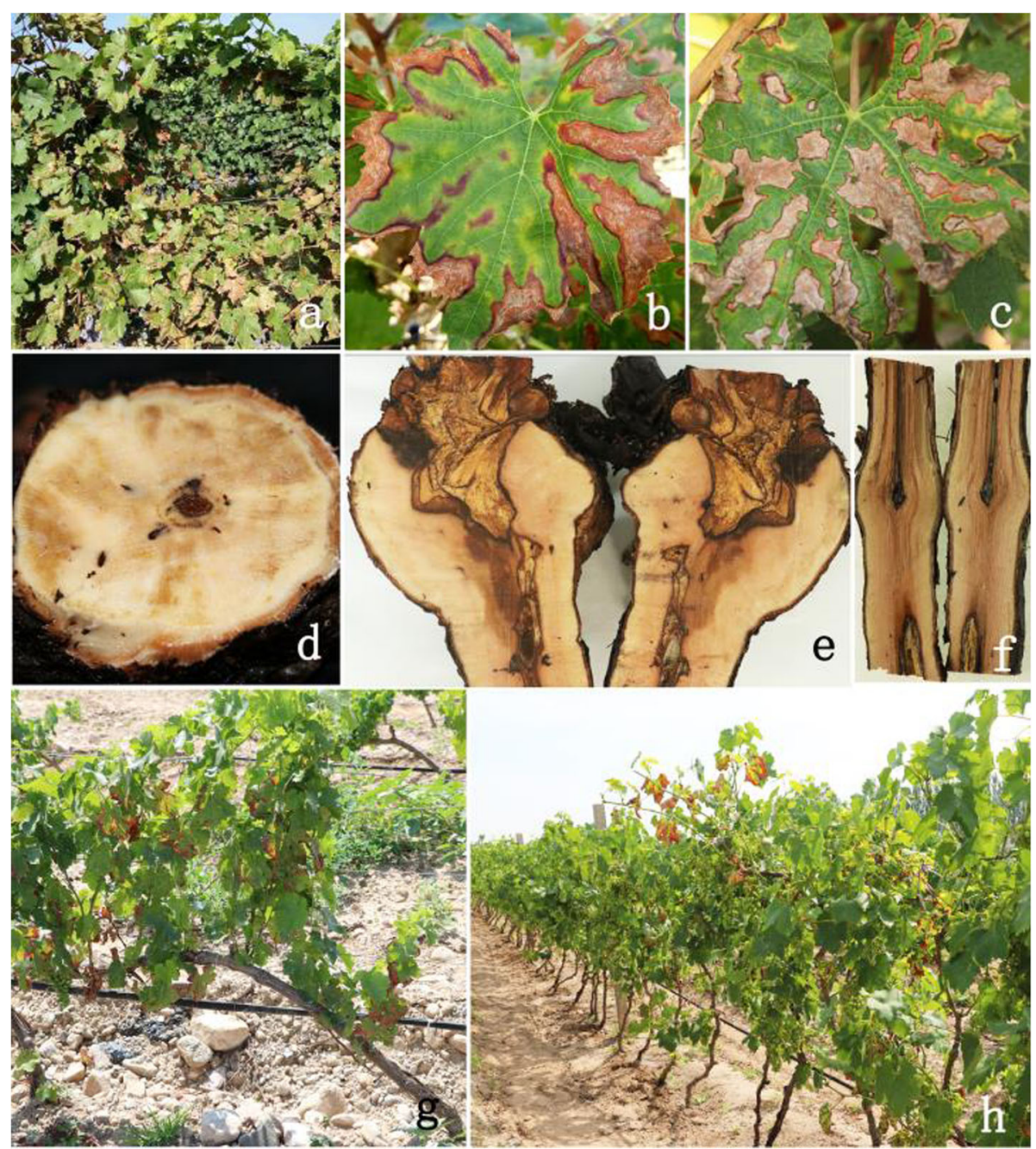

Fig. 1 The training systems in the surveyed vineyards and symptoms on 19-year-old 'Cabernet' grapevines. a-c Foliar symptoms of GLSD-like (Esca complex) disease, initially appeared as chlorotic spots and subsequently coalesced, turned dark red, and finally became necrotic. $\mathbf{d}-\mathbf{f}$ Internal symptoms of the same vine with foliar symptoms: discolored wood showing white rot and brown necrosis. $\mathbf{g}$, $\mathbf{h}$ The mini "J" and vertical training systems in the surveyed vineyards in Hebei and Ningxia provinces, China

JZB3190004, JZB3190006, JZB3190007, JZB3190009, JZB31900010, JZB31900011, JZB31900012) from this study, 54 reference strains, and one outgroup (Pleurostoma richardsiae CBS 270.33). The dataset consisted of 1796 total characters of which 310 were constant and 57 were parsimony-uninformative. A heuristic search of the remaining 788 parsimony-informative characters produced 1000 most parsimonious trees with a length of 1796 steps $(\mathrm{CI}=0.486, \mathrm{RI}=0.848, \mathrm{RCI}=0.412$ and $\mathrm{HI}=$ 0.514). Topology of the ML tree was similar to the MP tree. The best scoring RAxML tree with a final likelihood value of -8995.536137 is presented in Fig. 4. In this tree, isolates obtained in the present study clustered together with the $P$. minimum strains (including ex-type CBS 246.91) with $100 \%$ bootstrap values from both ML and MP analysis.
Phaeoacremonium minimum (Tul. \& C. Tul.) D. Gramaje, L. Mostert \& Crous in Gramaje et al. (2015) (Fig. 3).

Pathogenic on trunk and root of $V$. vinifera. Asexual morph: Conidia hyaline, ellipsoid to allantoid, 4.3-5.5 $\times$ $1.9-2.9 \mu \mathrm{m}$ (average $=4.9 \times 2.4 \mu \mathrm{m}, n=50$ ) (Fig. 3e). Conidiophores short and usually unbranched (Fig. 3f). Sexual morph: not observed.

Culture characteristics: Colonies on PDA reached $39.5 \pm 1.9 \mathrm{~mm}$ diameter after 21 days of incubation at $25^{\circ} \mathrm{C}$, with rare aerial mycelia and produced a yellow pigment on PDA (Fig. 3a, b). Mycelium texture was mostly verrucose.

Material examined: China, Hebei Province, Huailai County and Ningxia Province, Yinchuan, on trunk and root of $V$. vinifera, 10 August 2018, Qingtong Ye and 
Table 2 Isolates and GenBank accession numbers of Phaeoacremonium and Fomitiporia species in this study

\begin{tabular}{|c|c|c|c|c|c|c|}
\hline \multirow[t]{2}{*}{ Species } & \multirow[t]{2}{*}{ Strain } & \multicolumn{5}{|c|}{ GenBank accession number } \\
\hline & & $\overline{A C T}$ & TUB2 & LSU & ITS & EF1-a \\
\hline Phaeoacremonium africanum & CSN946 & KY906772 & KY906773 & N/A & N/A & N/A \\
\hline P. africanum & PMM2276 & KY906926 & KY906927 & N/A & N/A & N/A \\
\hline P. album & CBS 142688 & KY906884 & KY906885 & N/A & N/A & N/A \\
\hline P. album & CBS 142689 & KY906924 & KY906925 & N/A & N/A & N/A \\
\hline P. alvesii & CSN1239 & KY906784 & KY906785 & $\mathrm{N} / \mathrm{A}$ & N/A & N/A \\
\hline P. alvesii & CSN1335 & KY906800 & KY906801 & N/A & N/A & N/A \\
\hline P. aureum & CBS 142690 & KY906798 & KY906799 & $\mathrm{N} / \mathrm{A}$ & N/A & N/A \\
\hline P. aureum & CBS 142691 & KY906656 & KY906657 & N/A & N/A & N/A \\
\hline P. australiense & CSN490 & KY906728 & KY906729 & $\mathrm{N} / \mathrm{A}$ & $\mathrm{N} / \mathrm{A}$ & N/A \\
\hline P. australiense & CSN657 & KY906734 & KY906735 & N/A & N/A & N/A \\
\hline P. bibendum & CBS 142694 & KY906758 & KY906759 & N/A & $\mathrm{N} / \mathrm{A}$ & N/A \\
\hline P. fraxinopennsylvanicum & CSN66 & KY906680 & KY906681 & N/A & N/A & N/A \\
\hline P. gamsii & CBS 142712 & KY906740 & KY906741 & N/A & N/A & N/A \\
\hline P. geminum & CBS 142713 & KY906648 & KY906649 & N/A & N/A & N/A \\
\hline P. geminum & CBS 142717 & KY906646 & KY906647 & N/A & N/A & N/A \\
\hline P. globosum & CSN471 & KY906724 & KY906725 & N/A & N/A & N/A \\
\hline P. globosum & CSN1258 & KY906796 & KY906797 & N/A & N/A & N/A \\
\hline P. griseo-olivaceum & PMM1829 & KY906852 & KY906853 & N/A & N/A & N/A \\
\hline P. griseorubrum & PMM1828 & KY906850 & KY906851 & N/A & N/A & N/A \\
\hline P. griseorubrum & PMM1895 & KY906874 & KY906875 & N/A & N/A & N/A \\
\hline P. griseorubrum & PMM2220 & KY906896 & KY906897 & $\mathrm{N} / \mathrm{A}$ & N/A & N/A \\
\hline P. inflatipes & CSN47 & KY906664 & KY906665 & N/A & N/A & $\mathrm{N} / \mathrm{A}$ \\
\hline P. inflatipes & CSN57 & KY906674 & KY906675 & N/A & N/A & N/A \\
\hline P. iranianum & CSN170 & KY906694 & KY906695 & N/A & N/A & N/A \\
\hline P. iranianum & CSN267 & KY906706 & KY906707 & N/A & N/A & N/A \\
\hline P. italicum & CSN59 & KY906676 & KY906677 & N/A & N/A & N/A \\
\hline P. italicum & CSN119 & KY906690 & KY906691 & N/A & $\mathrm{N} / \mathrm{A}$ & N/A \\
\hline P. junior & CBS 142695 & KY906650 & KY906651 & N/A & N/A & N/A \\
\hline P. junior & CBS 142696 & KY906652 & KY906653 & N/A & $\mathrm{N} / \mathrm{A}$ & N/A \\
\hline P. longicollarum & CBS 142699 & KY906688 & KY906689 & N/A & N/A & N/A \\
\hline P. longicollarum & CBS 142700 & KY906878 & KY906879 & N/A & N/A & N/A \\
\hline P. meliae & CBS 142709 & KY906704 & KY906705 & N/A & N/A & N/A \\
\hline P. meliae & CBS 142710 & KY906824 & KY906825 & N/A & N/A & N/A \\
\hline P. minimum & CBS 246.91 & AY735497 & AF246811 & N/A & $\mathrm{N} / \mathrm{A}$ & N/A \\
\hline P. minimum & CBS 100397 & AY735498 & AF246806 & N/A & N/A & N/A \\
\hline P. minimum & PMM1305 & KY906836 & KY906837 & N/A & N/A & N/A \\
\hline P. minimum & PMM1323 & KY906842 & KY906843 & N/A & N/A & N/A \\
\hline P. minimum & PMM1822 & KY906846 & KY906847 & N/A & N/A & N/A \\
\hline P. minimum & PMM1967 & KY906886 & KY906887 & N/A & N/A & N/A \\
\hline P. minimum & JZB3190002 & MT294085 & МT294093 & N/A & N/A & N/A \\
\hline P. minimum & JZB3190004 & MT294086 & MT294094 & N/A & N/A & N/A \\
\hline P. minimum & JZB3190006 & MT294087 & MT294095 & N/A & N/A & N/A \\
\hline P. minimum & JZB3190007 & MT294088 & MT294096 & N/A & N/A & N/A \\
\hline
\end{tabular}


Table 2 Isolates and GenBank accession numbers of Phaeoacremonium and Fomitiporia species in this study (Continued)

\begin{tabular}{|c|c|c|c|c|c|c|}
\hline \multirow[t]{2}{*}{ Species } & \multirow[t]{2}{*}{ Strain } & \multicolumn{5}{|c|}{ GenBank accession number } \\
\hline & & ACT & TUB2 & LSU & ITS & EF1-a \\
\hline P. minimum & JZB3190009 & MT294089 & MT294097 & N/A & N/A & N/A \\
\hline P. minimum & JZB31900010 & MT294090 & MT294098 & N/A & N/A & N/A \\
\hline P. minimum & JZB31900011 & MT294091 & MT294099 & N/A & N/A & N/A \\
\hline P. minimum & JZB31900012 & MT294092 & MT294100 & N/A & N/A & N/A \\
\hline P. oleae & CBS 142701 & KY906718 & KY906719 & N/A & N/A & N/A \\
\hline P. oleae & CBS 142702 & KY906770 & KY906771 & N/A & N/A & N/A \\
\hline P. parasiticum & CSN24 & KY906658 & KY906659 & N/A & N/A & N/A \\
\hline P. parasiticum & CSN72 & KY906682 & KY906683 & N/A & N/A & N/A \\
\hline P. parasiticum & CSN79 & KY906686 & KY906687 & N/A & N/A & $\mathrm{N} / \mathrm{A}$ \\
\hline P. paululum & CBS 142705 & KY906880 & KY906881 & N/A & N/A & N/A \\
\hline P. proliferatum & CBS 142706 & KY906902 & KY906903 & N/A & N/A & N/A \\
\hline P. proliferatum & CBS 142707 & KY906826 & KY906827 & N/A & N/A & N/A \\
\hline P. prunicola & CSN398 & KY906716 & KY906717 & N/A & N/A & N/A \\
\hline P. prunicola & CSN719 & KY906752 & KY906753 & N/A & N/A & N/A \\
\hline P. rosicola & CBS 142708 & KY906830 & KY906831 & N/A & N/A & N/A \\
\hline P. scolyti & CSN27 & KY906660 & KY906661 & N/A & N/A & N/A \\
\hline P. scolyti & CSN55 & KY906670 & KY906671 & N/A & N/A & N/A \\
\hline P. sicilianum & CSN482 & KY906726 & KY906727 & N/A & N/A & N/A \\
\hline P. sicilianum & CSN930 & KY906768 & KY906769 & N/A & N/A & N/A \\
\hline P. spadicum & CBS 142711 & KY906838 & KY906839 & N/A & N/A & N/A \\
\hline P. spadicum & CBS 142714 & KY906666 & KY906667 & N/A & N/A & N/A \\
\hline P. subulatum & CSN42 & KY906662 & KY906663 & N/A & N/A & N/A \\
\hline P. subulatum & CSN51 & KY906668 & KY906669 & $\mathrm{N} / \mathrm{A}$ & N/A & $\mathrm{N} / \mathrm{A}$ \\
\hline$P$. venezuelense & PMM1138 & KY906834 & KY906835 & N/A & N/A & N/A \\
\hline P. viticola & CSN678 & KY906744 & KY906745 & N/A & N/A & N/A \\
\hline P. viticola & CSN701 & KY906748 & KY906749 & N/A & N/A & N/A \\
\hline Pleurostoma richardsiae & CBS 270.33 & AY579271 & AY579334 & N/A & N/A & N/A \\
\hline Fomitiporia aethiopica & MUCL 44777 & N/A & N/A & AY618204 & GU478341 & GU461893 \\
\hline F. aethiopica & MUCL 44806 & N/A & N/A & AY618202 & GU461944 & GU461892 \\
\hline F. alpina & Dai 15735 & N/A & N/A & KX639645 & KX639627 & KX639664 \\
\hline F. apiahyna & MUCL 51451 & N/A & N/A & GU461997 & GU461963 & GU461896 \\
\hline F. apiahyna & MUCL 51485 & N/A & N/A & GU461996 & GU461962 & GU461895 \\
\hline F. australiensis & MUCL 49406 & N/A & N/A & GU462001 & AY624997 & GU461897 \\
\hline F. baccharidis & MUCL 47756 & N/A & N/A & JQ087913 & JQ087886 & JQ087940 \\
\hline F. baccharidis & MUCL 47757 & N/A & N/A & JQ087914 & JQ087887 & JQ087941 \\
\hline F. bannaensis & MUCL 46926 & N/A & N/A & KF444705 & KF444682 & KF444751 \\
\hline F. bannaensis & MUCL 46930 & N/A & N/A & KF444706 & KF444683 & KF444752 \\
\hline F. bakeri & MUCL 51098 & N/A & N/A & JQ087901 & JQ087874 & JQ087928 \\
\hline F. calkinsii & MUCL 51099 & N/A & N/A & KF444709 & KF444686 & KF444755 \\
\hline F. calkinsii & MUCL 51398 & N/A & N/A & KF444710 & KF444687 & KF444756 \\
\hline F. capensis & MUCL 53009 & $\mathrm{~N} / \mathrm{A}$ & N/A & JQ087917 & JQ087890 & JQ087944 \\
\hline F. castilloi & MUCL 53481 & N/A & N/A & JQ087916 & JQ087889 & JQ087943 \\
\hline F. castilloi & MUCL 53980 & N/A & N/A & JX093830 & JX093786 & JX093743 \\
\hline
\end{tabular}


Table 2 Isolates and GenBank accession numbers of Phaeoacremonium and Fomitiporia species in this study (Continued)

\begin{tabular}{|c|c|c|c|c|c|c|}
\hline \multirow[t]{2}{*}{ Species } & \multirow[t]{2}{*}{ Strain } & \multicolumn{5}{|c|}{ GenBank accession number } \\
\hline & & ACT & TUB2 & LSU & ITS & EF1-a \\
\hline F. chilensis & CIEFAPCC 519 & N/A & $\mathrm{N} / \mathrm{A}$ & MK193750 & MK131089 & MK156786 \\
\hline F. chilensis & CIEFAPCC 587 & N/A & N/A & MK193752 & MK131094 & MK156789 \\
\hline F. cupressicola & MUCL 52489 & N/A & N/A & JQ087906 & JQ087879 & JQ087933 \\
\hline F. cupressicola & MUCL 52490 & $\mathrm{~N} / \mathrm{A}$ & $\mathrm{N} / \mathrm{A}$ & JQ087907 & JQ087880 & JQ087934 \\
\hline F. dryophila & MUCL 46380 & N/A & N/A & EF429219 & EF429238 & GU461900 \\
\hline F. dryophila & MUCL 51144 & N/A & N/A & KF444712 & KF444689 & KF444758 \\
\hline F. erecta & MUCL 49871 & N/A & $\mathrm{N} / \mathrm{A}$ & GU461976 & GU461939 & GU461903 \\
\hline F. expansa & MUCL 55026 & N/A & N/A & KJ401031 & KJ401032 & KJ401033 \\
\hline F. gaoligongensis & Cui 8261 & N/A & N/A & KX639642 & KX639624 & KX639663 \\
\hline F. gabonensis & MUCL 47576 & N/A & N/A & GU461990 & GU461971 & GU461923 \\
\hline F. gabonensis & MUCL 51291 & N/A & $\mathrm{N} / \mathrm{A}$ & GU461986 & GU461967 & GU461924 \\
\hline F. hainaniana & CL06-372 & N/A & N/A & KX639654 & KX663826 & KX639660 \\
\hline F. hartigii & MUCL 53550 & N/A & N/A & JX093832 & JX093788 & JX093745 \\
\hline F. hartigii & MUCL 53551 & N/A & N/A & JX093833 & JX093789 & JX093746 \\
\hline F. hippophaëicola & MUCL 31746 & N/A & $\mathrm{N} / \mathrm{A}$ & AY618207 & GU461945 & GU461904 \\
\hline F. hippophaëicola & MUCL 31747 & N/A & $\mathrm{N} / \mathrm{A}$ & GU461977 & GU461946 & GU461905 \\
\hline F. ivindoensis & MUCL 51311 & N/A & N/A & GU461979 & GU461952 & GU461907 \\
\hline F. ivindoensis & MUCL 51312 & N/A & N/A & GU461978 & GU461951 & GU461906 \\
\hline F. langloisii & MUCL 46375 & N/A & $\mathrm{N} / \mathrm{A}$ & EF429225 & EF429242 & GU461908 \\
\hline F. maxonii & MUCL 46017 & N/A & N/A & EF429230 & EF433559 & GU461910 \\
\hline F. maxonii & MUCL 46037 & N/A & N/A & EF429231 & EF433560 & GU461911 \\
\hline F. mediterranea & MCUL 38514 & N/A & N/A & AY618201 & GU461953 & GU461912 \\
\hline F. mediterranea & MCUL 45670 & N/A & N/A & GU461980 & GU461954 & GU461913 \\
\hline F. neotropica & MUCL 53114 & N/A & N/A & JX093836 & JX093792 & JX093749 \\
\hline F. nobilissima & MUCL 47580 & N/A & N/A & GU461985 & GU461966 & GU461921 \\
\hline F. nobilissima & MUCL 51289 & N/A & N/A & GU461984 & GU461965 & GU461920 \\
\hline F. norbulingka & Cui 9766 & N/A & N/A & KU364427 & KU364417 & KU364431 \\
\hline F. norbulingka & Cui 9777 & N/A & N/A & KU364428 & KU364418 & KU364432 \\
\hline F. pentaphylacis & Yuan 6012 & N/A & N/A & JQ003901 & JQ003900 & KX639671 \\
\hline F. polymorpha & MUCL 46166 & N/A & N/A & DQ122393 & GU461955 & GU461914 \\
\hline F. polymorpha & MUCL 46167 & N/A & $\mathrm{N} / \mathrm{A}$ & EF429233 & GU461956 & GU461915 \\
\hline F. pseudopunctata & MUCL 46168 & N/A & N/A & JQ087918 & JQ087891 & JQ087945 \\
\hline F. pseudopunctata & MUCL 51325 & N/A & N/A & GU461981 & GU461948 & GU461916 \\
\hline F. punctata & MUCL 53548 & N/A & $\mathrm{N} / \mathrm{A}$ & JX093834 & JX093790 & JX093747 \\
\hline F. punctata & Dai 15772 & N/A & N/A & KX639647 & KX639629 & KX639665 \\
\hline F. punicata & Dai 7175 & N/A & N/A & KX639650 & KX639632 & KX639668 \\
\hline F. punicata & Dai 10640 & N/A & N/A & KX639653 & KX663825 & KX639667 \\
\hline F. punicata & Cui 26 & N/A & N/A & GU461992 & GU461975 & GU461928 \\
\hline F. punicata & Cui 23 & N/A & N/A & GU461991 & GU461974 & GU461927 \\
\hline F. punicata & JZB3350001 & N/A & N/A & MT279704 & MT279702 & МТ304470 \\
\hline F. punicata & JZB3350002 & N/A & N/A & MT279705 & MT279703 & MT304471 \\
\hline F. robusta & MUCL 51297 & $\mathrm{~N} / \mathrm{A}$ & $\mathrm{N} / \mathrm{A}$ & JQ087919 & JQ087892 & JQ087946 \\
\hline F. robusta & MUCL 51327 & N/A & N/A & GU461993 & GU461949 & GU461929 \\
\hline
\end{tabular}


Table 2 Isolates and GenBank accession numbers of Phaeoacremonium and Fomitiporia species in this study (Continued)

\begin{tabular}{|c|c|c|c|c|c|c|}
\hline \multirow[t]{2}{*}{ Species } & \multirow[t]{2}{*}{ Strain } & \multicolumn{5}{|c|}{ GenBank accession number } \\
\hline & & $\overline{\mathrm{ACT}}$ & TUB2 & LSU & ITS & EF1-a \\
\hline F. sonorae & MUCL 47689 & N/A & N/A & JQ087920 & JQ087893 & JQ087947 \\
\hline F. subhippophaëicola & Cui 12096 & N/A & N/A & KU364426 & KU364421 & KU364437 \\
\hline F. subhippophaëicola & Cui 12102 & N/A & N/A & KU364424 & KU364423 & KU364435 \\
\hline F. subrobusta & Dai 13576 & N/A & N/A & KX639635 & KX639617 & KX639655 \\
\hline F. subrobusta & Dai 13577 & N/A & N/A & KX639636 & KX639618 & KX639656 \\
\hline F. tabaquilio & MUCL 46230 & $\mathrm{~N} / \mathrm{A}$ & N/A & DQ122394 & GU461940 & GU461931 \\
\hline F. tabaquilio & MUCL 47754 & N/A & N/A & GU461994 & GU461941 & GU461932 \\
\hline F. tenuis & MUCL 44802 & N/A & N/A & AY618206 & GU461957 & GU461934 \\
\hline F. tenuis & MUCL 49948 & N/A & N/A & GU461998 & GU461958 & GU461935 \\
\hline F. tenuitubus & Dai 16204 & N/A & N/A & KX639637 & KX639619 & KX639657 \\
\hline F. tenuitubus & Yuan 5736 & N/A & N/A & JQ003903 & JQ003902 & KX639658 \\
\hline F. texana & MUCL 47690 & N/A & N/A & JQ087921 & JQ087894 & JQ087948 \\
\hline F. texana & MUCL 51143 & N/A & N/A & JQ087922 & JQ087895 & JQ087949 \\
\hline F. torreyae & Dai 15517 & N/A & N/A & KX639651 & KX639633 & KX639669 \\
\hline F. torreyae & Dai 15521 & N/A & N/A & KX639652 & KX639634 & KX639670 \\
\hline F. torreyae & WC31 & N/A & N/A & JQ087924 & JQ087897 & JQ087951 \\
\hline F. tsugina & MUCL 52702 & N/A & N/A & JQ087925 & JQ087898 & JQ087952 \\
\hline F. tsugina & MUCL 52703 & N/A & N/A & JQ087926 & JQ087899 & JQ087953 \\
\hline Phellinus uncisetus & MUCL 46231 & N/A & N/A & EF429235 & GU461960 & GU461937 \\
\hline P. uncisetus & MUCL 47061 & N/A & N/A & GU462000 & GU461972 & GU461938 \\
\hline
\end{tabular}

CBS: Westerdijk Fungal Biodiversity Institute, Utrecht, Netherlands; JZB: Beijing Academy of Agriculture and Forestry Sciences Culture Collection, China; PMM: Collection of Providence Moyo at the University of Stellenbosch Department of Plant Pathology, Stellenbosch, South Africa; MUCL: Mycothèque de I'Université catholique de Louvain

Ex-type is in bold, and the newly generated sequences in present study are in italics

$\mathrm{N} / \mathrm{A}$ : the sequence is not available or not applicable to the present study

Xinghong Li, living cultures JZB3190002, JZB3190004, JZB3190006, JZB3190007, JZB3190009, JZB31900010, JZB31900011 and JZB31900012.

\section{Pathogenicity \\ Pathogenicity of F. punicata}

Sixty days after inoculation, 5 of the 20 plants inoculated with $F$. punicata showed chlorotic leaf spots that gradually coalesced. Three months post-inoculation, in control plants, there were neither leaf symptoms nor any significant discoloration in the wood (Fig. 5a-e). While in the vines inoculated with $F$. punicata, the interveinal discolorations on leaves turned dark red and finally became necrotic, and internal wood discolorations were also observed: brown discoloured lesions spread up and down from the inoculation sites in the trunk (Fig. $5 f-j$ ), but no white rot symptoms were found in the wood. $F$. punicata induced wood discolorations with a mean length of $8.2 \pm 1.1 \mathrm{~mm}$. The inoculated fungus was reisolated and confirmed as F. punicata, with an $80 \%$ isolation ratio. No fungal pathogens were isolated from control plants.

\section{Pathogenicity of $P$. minimum}

At 108 days post-inoculation (dpi), leaves of three out of ten vine cuttings inoculated with the $P$. minimum isolate JZB3190002 displayed necrotic lesions starting from the edge of the leaf blade, which initially appeared as chlorotic spots subsequently coalesced along the veins. At $208 \mathrm{dpi}$, no discolouration or necrosis was recorded in the control vines (Fig. 6a-f), while three inoculated vines showed a clear interveinal discoloration and necrosis on leaves, which resembled the tiger-like symptom. Analysis of the internal wood symptoms revealed the development of brown necrosis in the stem starting from the base (Fig. $6 \mathrm{~g}-\mathrm{l}$ ) of the three symptomatic inoculated vines. The pathogen was re-isolated and confirmed as $P$. mimimum, with $60 \%$ isolation ratio. No fungal species were isolated from control plants.

\section{Discussion}

GLSD, one of the diseases within the Esca complex was observed in our surveys. The most prevalent external symptoms of the disease were tiger stripe pattern on leaves. All the symptomatic vines (with typical foliar 


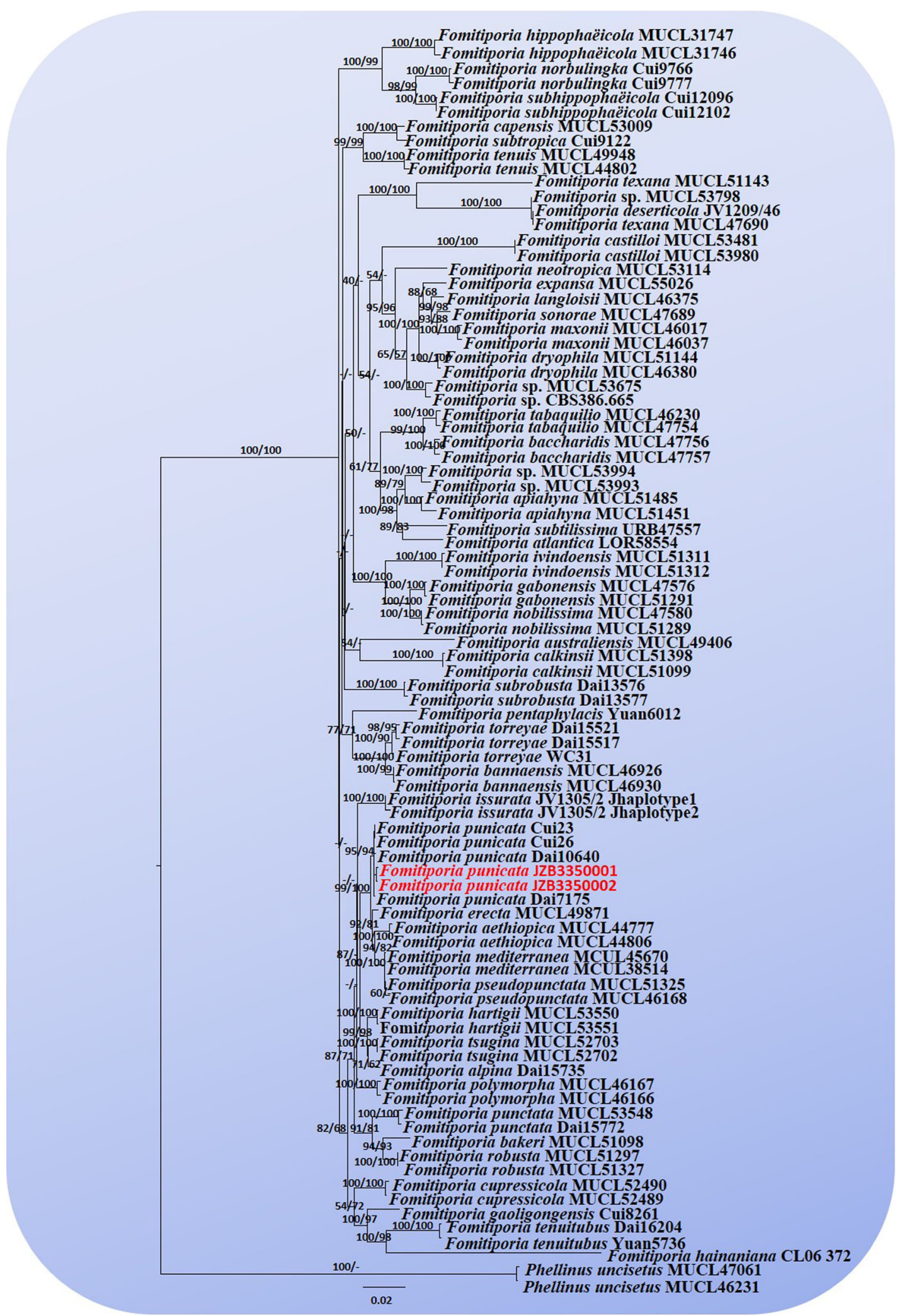

Fig. 2 RAXML tree obtained from phylogenetic analysis of combined genes of LSU, ITS and EF1-a. Maximum likelihood and parsimony bootstrap values are showed in nodes (the values lower than $50 \%$ are not given). The scale bar shows 0.02 changes. The tree is rooted in Phellinus uncisetus (MUCL 47061 and MUCL 46231) 


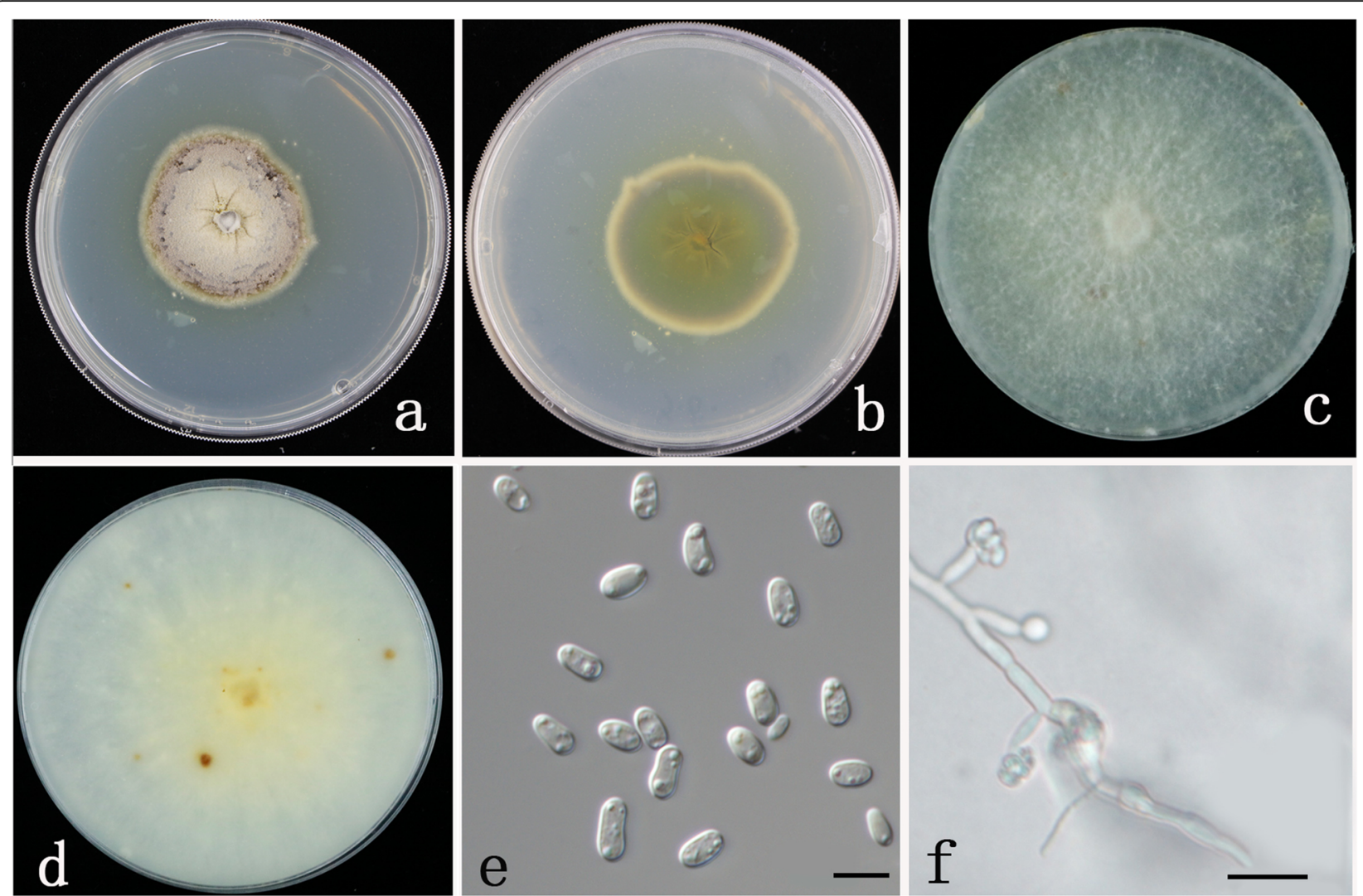

Fig. 3 Fungi isolation and morphological characterization. $\mathbf{a}, \mathbf{b}$ Phaeoacremonium minimum grown on PDA media for 14 days. The front (a) and reverse (b) sides of the culture plate. $\mathbf{c}$, $\mathbf{d}$ Fomitiporia punicata grown on PDA media for 18 days. The front (c) and reverse (d) sides of the culture plate. $\mathbf{e}, \mathbf{f}$ Conidia and conidiogenous cells of $P$. minimum. Bar $=10 \mu \mathrm{m}$ in (e); Bar $=20 \mu \mathrm{m}$ in (f)

symptoms) that were checked for internal wood symptoms showed discoloration and necrosis in wood tissues. The white rot wood symptom was found at the base of trunk and rootstock of only one adult grapevine (19year-old), which was consistent with previous studies that white rot symptom were frequently found in old vineyards (Mugnai et al. 1999; Mondello et al. 2018). No signs of black measles and apoplexy were found during the survey. The disease incidence of Esca complex varied with cultivar, vineyard and year, confirming the symptoms fluctuation as a typical phenomenon in this disease (Surico et al. 2006). This fluctuation is also known to be affected by the vineyard age, amount of rainfall in the rainy seasons (Surico et al. 2000) together with other environmental factors (Surico et al. 2006). During the surveys, we discovered the reason why there have been no reports of Esca complex in China up to now. Usually, the grape growers do not realize that the tiger-striped foliar symptoms are caused by biotic agents and they treat them as if they were a nutrient deficiency. To our knowledge, it is possible for the vascular pathogens to be introduced with infected propagation materials from abroad, whereas the decay causing agents usually do not move with propagation materials, but instead they contaminate the pruning wounds on vines. The wood decay agents reported here have never been recorded outside China before.

In our study, morphological characterization combined with multigene-combined phylogenetic analyses demonstrated that the prevalent isolates that are associated with wood symptoms are $F$. punicata and $P$. minimum. $P$. minimum was found in both Hebei and Ningxia provinces, while F. punicata was detected only in Hebei Province. Pathogenicity tests confirmed that both of these two fungal species were able to cause tiger stripe symptoms on leaves.

Fomitiporia species associated with Esca complex were found to vary with geographic location (Cloete 2015). Fomitiporia mediterranea has been reported mainly in European and Mediterranean grape-growing regions (Larignon and Dubos 1997; Fischer 2002; Luque et al. 2009; Akgul et al. 2015; Baranek et al. 2018), F. polymorpha in North America (Fischer 2006), F. australiensis in Australia (Fischer 2006) and F. capensis in South Africa (Cloete et al. 2014). Fomitiporia punicata was first reported on Punica granatum (Punicaceae) by Dai et al. 


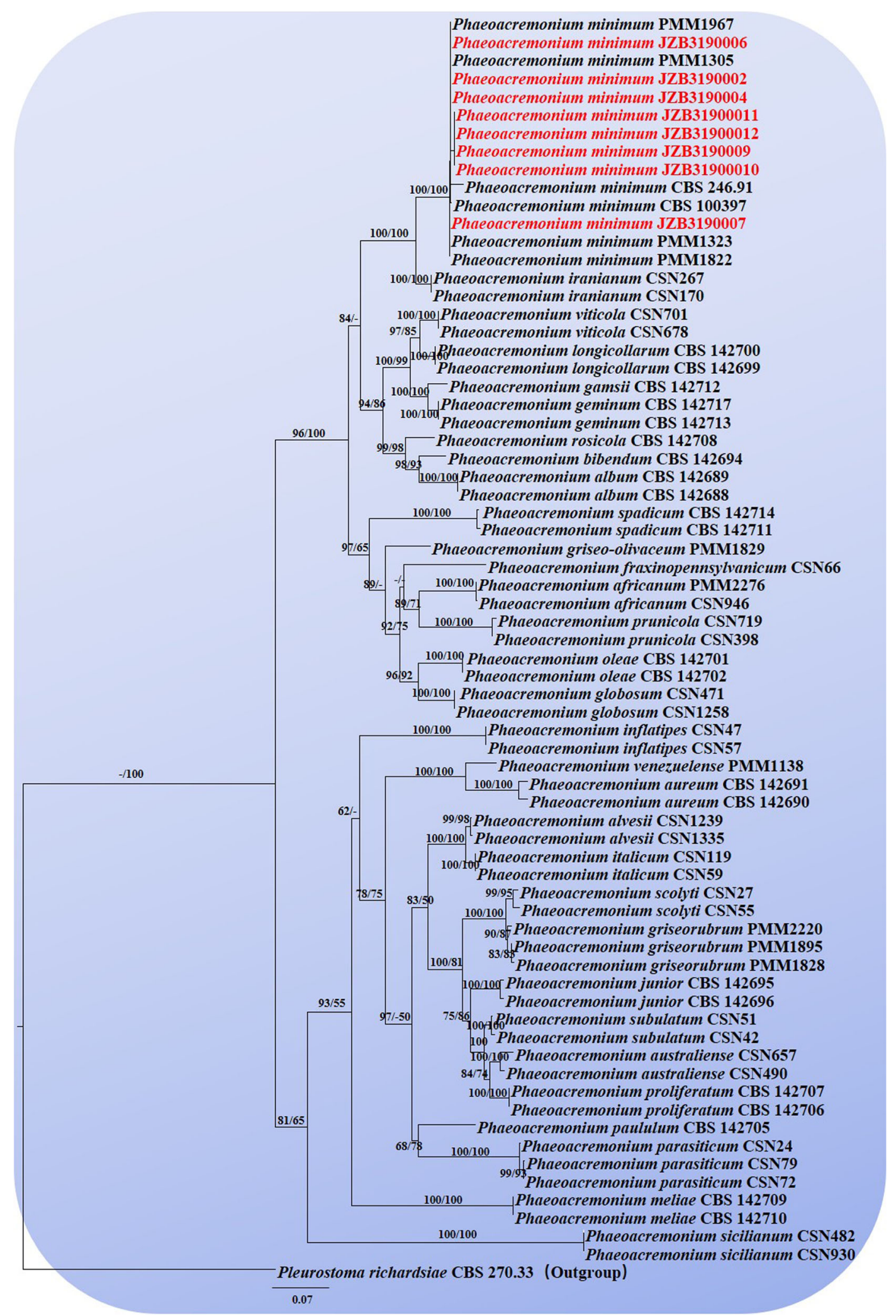

Fig. 4 RAXML tree obtained from phylogenetic analysis of combined genes of actin and beta-tubulin. Maximum likelihood and parsimony bootstrap values are shown in nodes (the values lower than $50 \%$ are not given). The scale bar shows 0.07 changes. The tree is rooted in Pleurostoma richardsiae (CBS 270.33) 


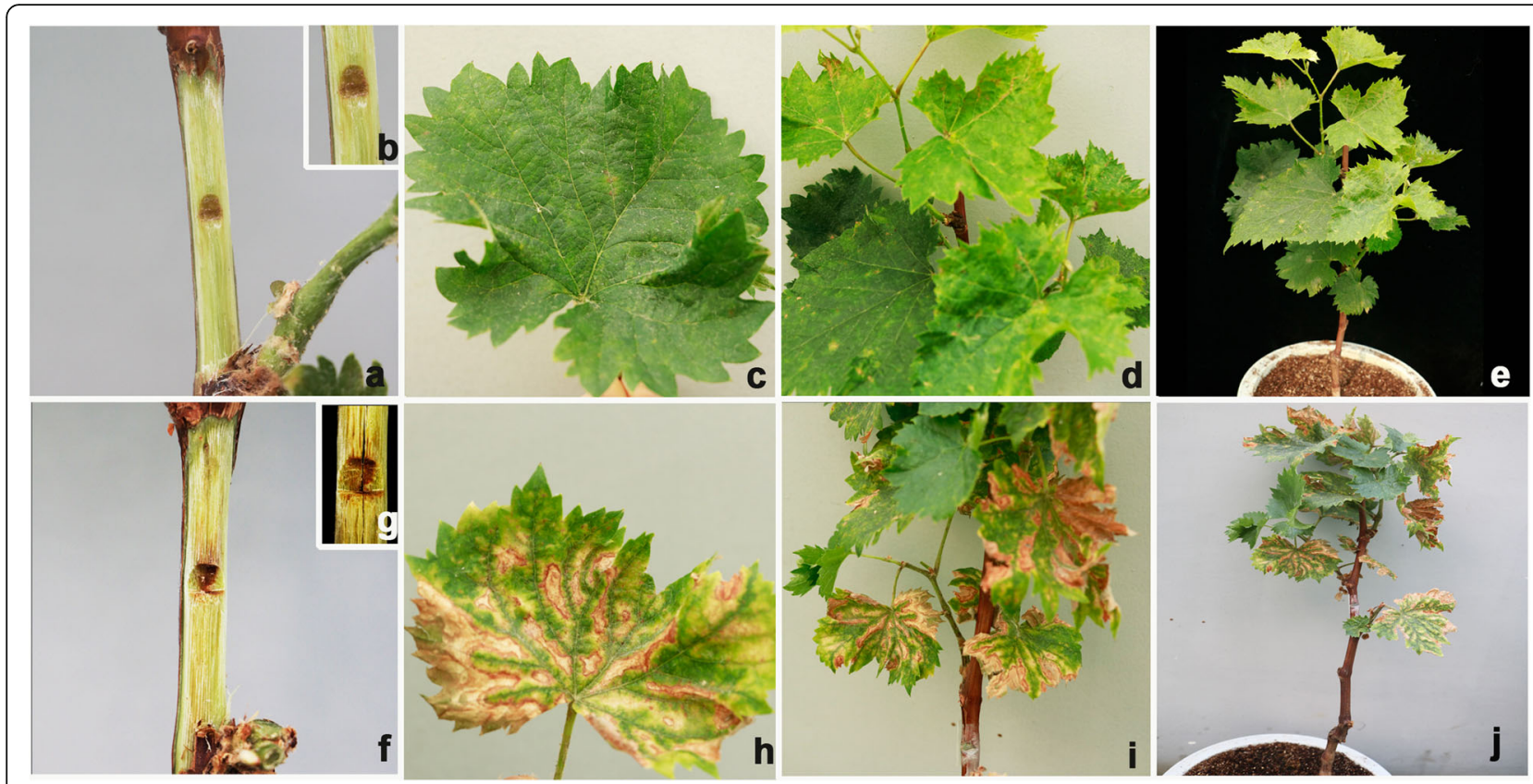

Fig. 5 Pathogenicity test of Fomitiporia punicata in the greenhouse. a-e Control plant. $\mathbf{f}-\mathbf{j}$ The plant (cv. Cabernet, 2-year-old) inoculated with $F$. punicata showed interveinal discolorations and necrosis on leaves and black necrosis within the trunk at 3 months post-inoculation

(2008), and this is the first report of $F$. punicata infecting grapevines and causing Esca complex disease. In China, the fruiting bodies of F. punicata were found on living trees of Punica granatum in Shanxi Province (Dai et al. 2008). Although we isolated this fungus from diseased grapevines in this study, we did not find any fruiting bodies. It was also indicated in previous study that the fruiting bodies of basidiomycete species associated with Esca complex are rarely formed and observed on grapevines (Cloete et al. 2014).

Several genera of vascular pathogens, like Phaeoacremonium, Phaeomoniella and Cadophora, and

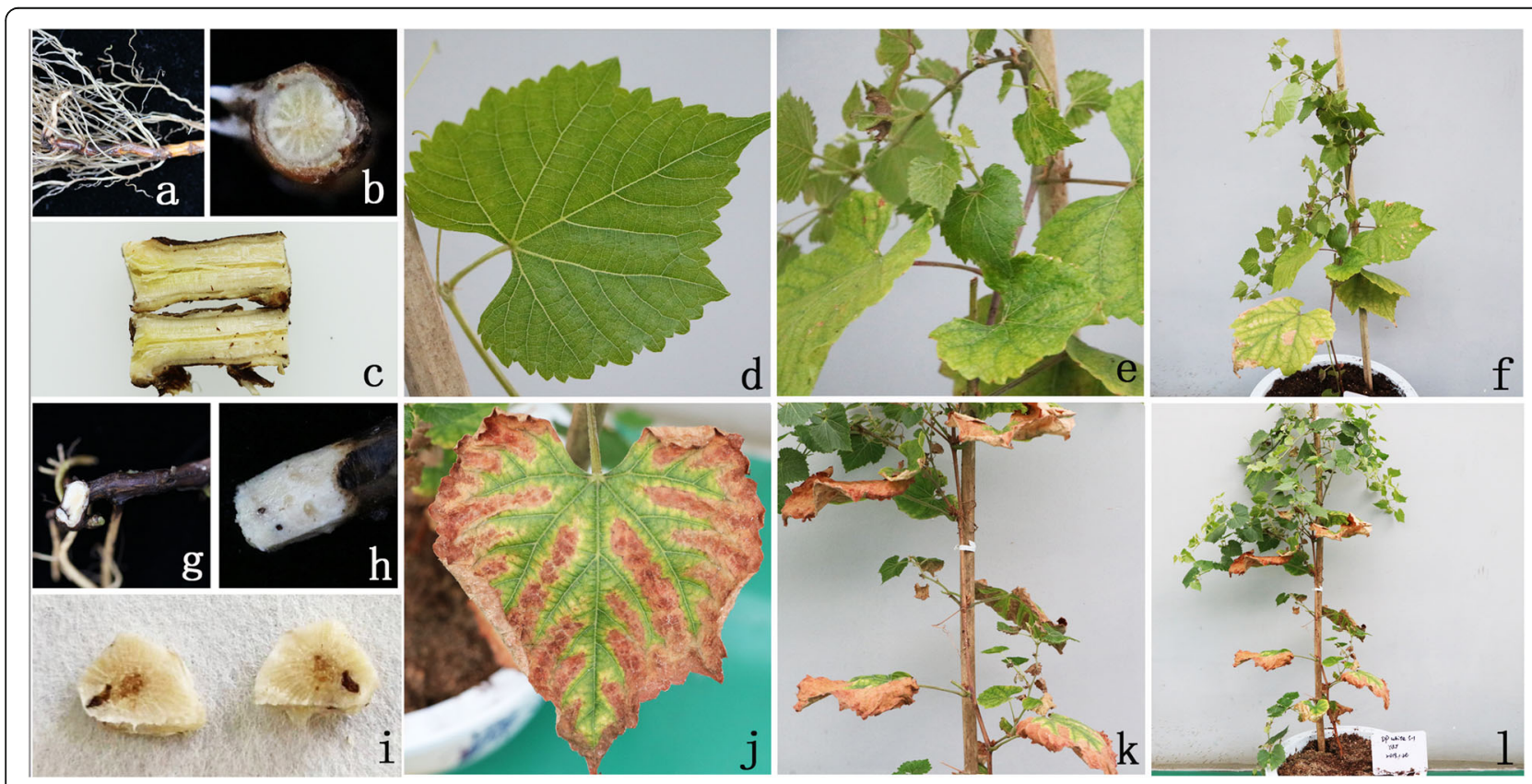

Fig. 6 Pathogenicity test of Phaeoacremonium minimum in the greenhouse. a-f Control plant. $\mathbf{g}-\mathbf{I}$ The Plant inoculated with P. minimum showed interveinal discolorations and necrosis on leaves, and black necrosis within rootstock at $208 \mathrm{dpi}$ 
basidiomycete species belonging to the genera Inocutis, Inonotus, Fomitiporella, Fomitiporia, Phellinus and Stereum, have been reported on grapevine worldwide (Fischer 2006; Cloete 2015). Of these, P. minimum is one of the most frequently reported species while Phaeomoniella chlamydospora is the most virulent species (Gramaje et al. 2018). Phaeoacremonium minimum has been reported on a wide range of woody hosts, primarily on Vitis species (Gramaje et al. 2015; da Silva et al. 2017; Spies et al. 2018). It also has a wide distribution and has been reported in Algeria, Argentina, Australia, Brazil, California, Chile, China, France, Iran, Italy, South Africa, Spain, Uruguay, Turkey and the USA (Crous et al. 1996; Larignon and Dubos 1997; Scheck et al. 1998; Gatica et al. 2001; Groenewald et al. 2001; Edwards and Pascoe 2004; Gimenez-Jaime et al. 2006; Mostert et al. 2006; Abreo et al. 2011; Berraf-Tebbal et al. 2011; Úrbez-Torres et al. 2012; Correia et al. 2013; Mohammadi et al. 2013; Ye et al. 2020).

Other fungi causing Esca complex include species in the genera Neofusicoccum and Diaporthe. Neofusicoccum and Diaporthe species are also the causal organisms of GTDs (Yan et al. 2013; Dissanayake et al. 2015a). Diplodia seriata and Eutypa lata have been isolated from Esca-affected vines in Europe (Mugnai et al. 1999). Therefore, it is possible to isolate the pathogens causing Esca complex and Botryosphaeria dieback from the same grapevine during the growing season in China. The results also provide evidence that in China GTDs are associated with a wide range of fungal taxa.

As shown in the present study, the Esca complex symptoms in China are also associated with rootstock infection, thus removing the whole infected plants is a very critical control measure currently available to inhibit the spread of this disease. Moreover, frequent and extensive pruning or large rejuvenation cuttings are known to be favourable for the development of Esca disease (Mugnai et al. 1999). Extensive wounds are normally created in summer and winter trimming; thus, protection of pruning wounds is an essential measure to prevent the spread of the disease in the vineyards. Further intensive field surveys are required in all grapegrowing regions in China to understand the extent and severity of these diseases.

\section{Conclusions}

Here we provide the first detailed study of the presence of Esca complex of grapevine in China. We also present the first record of $F$. punicata associated with Esca complex of grapevine worldwide and confirm the presence of $P$. minimum in the same vines infected with $F$. punicata. Further studies need to be carried out to understand the impact of this disease complex in China.

\section{Methods}

\section{Vineyard surveys and sample collection}

Hebei and Ningxia provinces are two major wine and table grape-growing regions in China (Chen 2018). The climate of Hebei and Ningxia provinces is temperate monsoon climate (Yan et al. 2013). The soil type in surveyed vineyards of Hebei Province is mainly sandy soils (Wang 2012), and gravel calcareous soils in Ningxia Province (Wang 2018). The average altitude of vineyards we investigated in Hebei and Ningxia provinces is about $1300-1600$ and $1000-1500$ m.a.s.l., respectively. The annual rainfall in Ningxia Province does not exceed 200 $\mathrm{mm}$. The sunshine, soil, moisture, altitude and latitude at the Helan Mountain's East Foothill are conducive to grape cultivation.

Surveys of Esca complex symptoms were carried out in Hebei $\left(36^{\circ} 01^{\prime}-42^{\circ} 37^{\prime} \mathrm{N}, 113^{\circ} 04^{\prime}-119^{\circ} 53^{\prime} \mathrm{E}\right)$ and

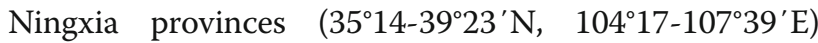
from 2017 to 2019. At least one cultivar was investigated in each vineyard. Disease incidence was calculated by counting the plants in a block of grapevines in each vineyard that showed foliar symptoms among the total number of standing vines surveyed. The surveyed vineyards varied from 5 to 20 -year-old. The training system applied in the surveyed vineyards were mini "J" and, in some vineyards in the two provinces of China were vertical training system (Fig. 1g, h). Based on the information obtained from the winegrowers, the original propagation materials in vineyards $\mathrm{Hb}-1, \mathrm{Hb}-2, \mathrm{Hb}-3$ and Nx-1 were bought from France. The surveyed grape cultivars are Marselan, Chardonnay, Merlot, Petit Verdot, Cabernet Franc, and there were some unknown varieties as well. Some grapevines were grafted but the rootstock is unknown, and some were self-rooted.

GLSD foliar symptoms (Esca complex) were recorded by the grape growers in some vineyards, which are included in Table 1 . The yearly variation in incidence of GLSD foliar symptoms in different regions and cultivars was recorded. Characteristic symptoms, such as tiger striped leaves and wood discoloration, were recorded and photographed. Samples from symptomatic trunk and rootstock of 12 plants were collected and taken to the laboratory for further study.

\section{Fungal isolation and morphological characterization}

Portions of scion trunks and rootstocks were put in zip-lock bags and kept at $4{ }^{\circ} \mathrm{C}$ for further studies. Samples were analysed and different types of wood symptoms and discolorations were detected, which included white rot, brown necrosis, brown to black streaks appearing as black spots in cross-section, black line bordering the decayed wood, decayed pith, pith margin, asymptomatic scion trunk base and rootstock and asymptomatic tissues (no discoloration or 
decay). Pathogens were isolated by dissecting small wood pieces $\left(<5 \mathrm{~mm}^{2}\right)$ after taking off the bark. Wood chips were taken from symptomatic woods with discoloured, necrotic or decayed symptoms, disinfected in $1.5 \%$ sodium hypochlorite for $3 \mathrm{~min}$, in $70 \%$ ethanol for $30 \mathrm{~s}$, and then rinsed three times with sterile distilled water, dried and finally placed on potato dextrose agar (PDA) amended with ampicillin $(100 \mathrm{mg} / \mathrm{L})$. The plates were incubated at $25^{\circ} \mathrm{C}$ in the dark. The wood pieces without any hyphal development were transferred onto new PDA plates to avoid contamination by fast-growing fungi from the closely placed wood pieces (Hofstetter et al. 2012). Hyphal tips from actively growing margins of the colony were transferred onto new PDA plates. From morphologically identical colonies, a few strains were selected for further analysis. To obtained pure cultures, single spore isolations were made on PDA plates. Conidia were diluted with sterile distilled water and spread on $1.5 \%$ water agar (WA), and incubated at $25^{\circ} \mathrm{C}$ and germinating single conidia were transferred to PDA plates. For those isolates that did not produce conidia, hyphal tip isolation was performed to obtain pure cultures. Cultures were examined periodically for the development of conidiomata. Colony diameters after 5-7 days of incubation were measured. The shapes, sizes, and colour of at least 50 conidia were observed and recorded using ZENPRO 2012 software (Carl Zeiss Microscopy). All the cultures obtained in this study were deposited in the culture collection of Institute of Plant and Environment Protection, Beijing Academy of Agriculture and Forestry Sciences (JZB), Beijing, China.

\section{DNA extraction, PCR amplification and sequence assembly}

All isolates were initially identified by morphological characteristics, including colony morphology and conidial characters. At least two isolates were selected from each morphologically similar group for molecular identification. In the present study, colony characters of all the Basidiomycete isolates were similar on PDA, therefore only two isolates (JZB3350001 and JZB3350002) were selected for the phylogenetic analyses. Colonies of the Phaeoacremonium isolates differed on PDA at $25^{\circ} \mathrm{C}$ in dark, so eight isolates (JZB3190002, JZB3190004, JZB3190006, JZB3190007, JZB3190009 JZB31900010, JZB31900011, JZB31900012) representing different colony morphological groups were selected. Total genomic DNA was extracted from 50 to $100 \mathrm{mg}$ of mycelia, scraped from 7-day-old colonies on PDA, by the CTAB method (Guo et al. 2000). For preliminary species identification, the internal transcribed spacer (ITS) region was amplified with the primer pair ITS1/ITS4 (White et al.
1990). The sequences obtained were used to search GenBank with the BLASTn tool (https://blast.ncbi.nlm.nih. gov/Blast.cgi). Based on BLAST results, additional gene fragments of $\beta$-tubulin (tub2) and actin (act) were amplified and sequenced for Phaeoacremonium isolates with primer pairs T1/ Bt2b (Glass and Donaldson 1995; O'Donnell and Cigelnik 1997) and ACT-512F/ACT783R (Carbone and Kohn 1999), respectively. For the basidiomycete isolates, nuclear large subunit ribosomal RNA gene regions (nLSU) and elongation factor- $1 \alpha$ (tef1) gene fragments were amplified and sequenced with two primer pairs LROR/ LR7 (http://www.biology.duke. edu/fungi/mycolab/primers.htm) and EF-983F/ EF1567R (http://ocid.NACSE.ORG/research/deephyphae/ EF1primer.pdf), respectively. The PCR mixture consisted of $1 \mu \mathrm{L}$ of genomic DNA, $2 \mathrm{mM} \mathrm{MgCl}_{2}, 10 \mu \mathrm{M}$ of each deoxyribonucleotide triphosphates (dNTPs), $1 \mu \mathrm{L}$ of each $10 \mu \mathrm{M}$ primer and $0.3 \mathrm{U}$ of Taq Silver star DNA polymerase in a $1 \times$ reaction buffer. The PCR conditions were as follows: for tub2 and tef1 amplification, initial denaturation for $3 \mathrm{~min}$ at $94^{\circ} \mathrm{C}, 34$ cycles of denaturation at $94{ }^{\circ} \mathrm{C}$ for $30 \mathrm{~s}$, annealing for $30 \mathrm{~s}$ at $56^{\circ} \mathrm{C}$, and extension for $30 \mathrm{~s}$ at $72^{\circ} \mathrm{C}$, and a final extension for $7 \mathrm{~min}$ at $72^{\circ} \mathrm{C}$; for act and ITS, the same as for tub2 except for annealing at $54^{\circ} \mathrm{C}$; for nLSU, the same as for tub2 except for extension for $90 \mathrm{~s}$ at $72{ }^{\circ} \mathrm{C}$. PCR products were separated on $1 \%$ agarose gels and visualized under UV light in a Gel DocTM XR Molecular Imager (Bio-Rad, USA). Sequencing was carried out by Tsingke Company, Beijing, China. Sequence quality was confirmed by checking chromatograms with BioEdit v.5 (Hall 1999). For sequences obtained from forward and reverse primers, consensus sequences were generated with SeqMan 3.1 (DNASTAR, Inc.). New sequences obtained in the present study were deposited in GenBank database (Table 2).

\section{Phylogenetic analyses}

Reference sequences of Fomitiporia and Phaeoacremonium reference species and related taxa were obtained from GenBank (Groenewald et al. 2001; Chen and Cui 2017; Spies et al. 2018), which were aligned with the sequences obtained in the present study using MAFFT (Kuraku et al. 2013; Katoh et al. 2019). The alignments were edited manually with BioEdit v7.0.9 (Hall 1999) where necessary. Phylogenetic relationships were inferred by maximum parsimony (MP) implemented in PAUP (v4.0) (Swofford 2002), and maximum likelihood (ML) in RAxML (Silvestro and Michalak 2012). In maximum parsimony, heuristic searches with tree-bisectionreconnection (TBR), as the branch-swapping algorithm and 1000 bootstrap replications were used with random sequence addition. All characters were unordered and carried equal weight, gaps were treated as missing data, 
Steepest descent option not in effect and MulTrees option in effect. Descriptive tree statistics including Tree length (TL), Consistency index (CI), Retention index (RI), Relative consistency index (RCI) and Homoplasy index (HI) were calculated. Resulting trees were assessed by the Kishino-Hasegawa tests to determine whether they were significantly different or not (Kishino and Hasegawa 1989). The ML analysis was performed with RAxML-HPC2 on XSEDE (8.2.8) (Stamatakis 2014) in the CIPRES Science Gateway Platform (Miller et al. 2010). For each phylogenetic tree, 1000 nonparametric bootstrapping iterations were used. Phylogenetic trees were visualized with FigTree v1.4.4 (Rambaut 2018) and edited with Microsoft PowerPoint 2016.

\section{Pathogenicity tests}

\section{Pathogenicity test for $F$. punicata}

Young, healthy, two-year-old 'Cabernet Franc' rooted cuttings in pots were used for the pathogenicity tests. The grapevines stems were first surface sterilized with $70 \%$ ethanol, then 5-mm-diameter wounds were made on the stem using a sterilized blade. One wound was made on each plant. Mycelial plugs (5-mm-diameter) taken from actively growing colony margins of $F$. punicata (JZB3350001 and JZB3350002 obtained in this study) were placed on the wounds. Wounds were covered with Vaseline (petroleum jelly) and sealed with parafilm to prevent water evaporation. Control plants were inoculated with non-colonized PDA plugs. For each isolate, the experiment was replicated on 10 plants. Inoculated plants were grown under greenhouse conditions $\left(24-25^{\circ} \mathrm{C}, 60 \% \mathrm{RH}\right)$ for more than three months until they showed symptoms. When tiger stripe symptoms appeared on the leaves, inoculated and control young vines were analysed for development of wood symptoms. To confirm Koch's postulate, the target pathogen was reisolated from the inoculated vines.

\section{Pathogenicity test for Phaeoacremonium minimum}

Young, healthy, two-month-old 'Furcal' grapevine cuttings propagated from vines grown in greenhouse were used for the pathogenicity tests. Two isolates obtained in this study (JZB3190002 and JZB3190004) were selected for inoculation. Roots and base of stems were cleaned in running tap water, then surface sterilized with $70 \%$ ethanol for $5 \mathrm{~s}$ and finally rinsed with sterile distilled water three times. The ends of the sterilized roots were wounded by cutting off the terminal $1 \mathrm{~cm}$ with sterilized scissors. The roots were then dipped in spore suspension $\left(10^{6}\right.$ spores $\left./ \mathrm{mL}\right)$ for $5 \mathrm{~min}$. After that, the plants were transferred into pots $(20.5 \mathrm{~cm}$ in diameter, $2 \mathrm{~L})$ with moist soil and $25 \mathrm{~mL}$ of spore suspension was added to the soil around the base of stem. Control plants were immersed in sterile water for the same period of time and planted in pots with soil. For each isolate, the experiment was replicated on 10 plants. Inoculated plants were grown under greenhouse conditions (24$\left.25^{\circ} \mathrm{C}, 60 \% \mathrm{RH}\right)$ for more than three months until they showed foliar symptoms. Once foliar symptoms were developed, the pathogen was re-isolated from the necrotic wood tissues on inoculated or control plants and identified according to cultural and morphological characters.

\begin{abstract}
Abbreviations
Cl: Consistency index; F. punicata: Fomitiporia punicata; GLSD: Grapevine leaf stripe disease; GTDs: Grapevine trunk diseases; HI: Homoplasy index; ITS: Internal transcribed spacer; MEA: Malt extract agar; ML: Maximum likelihood; MP: Maximum parsimony; OIV: International Organization of Vine and Wine; P. minimum: Phaeoacremonium minimum; PDA: Potato dextrose agar; RCl: Relative consistency index; Rl: Retention index; TBR: Tree-bisectionreconnection; TL: Tree length; WA: Water agar
\end{abstract}

\section{Supplementary Information}

The online version contains supplementary material available at https://doi. org/10.1186/s42483-021-00087-w.

Additional file 1: Table S1. Symptom types in vine woods detected in 12 vines. Table S2. Correlation between wood symptoms and fungal species

\section{Acknowledgements \\ We are thankful to Prof. Yafeng Dong (Research Institute of Pomology, Chinese Academy of Agricultural Sciences) for providing grapevines Furcal and Cabernet Franc. We also thanks to Dr. Alan John Lander Phillips (Biosystems and Integrative Sciences Institute, University of Lisbon) for helping us to revise the manuscript.}

\section{Authors' contributions}

JY designed the research and revised the manuscript; QY performed the research and wrote the manuscript; JJ performed the pathogenicity test of Phaeoacremonium minimum and took photographs of the symptoms. ISM revised the manuscript and provided the plant materials. $X L$ and WZ collected some Esca-diseased samples and revised the manuscript. LM gave some ideas and deeply revised the manuscript. $\mathrm{KDH}$ and $\mathrm{XW}$ gave some comments and revised the manuscript. All authors read and approved the final manuscript.

\section{Funding}

This study was funded by Beijing Talent Program for Dr. Jiye Yan and the China Agriculture Research System (CARS-29).

Availability of data and materials

Not applicable.

\section{Declarations}

Ethics approval and consent to participate Not applicable.

\section{Consent for publication \\ Not applicable.}

\section{Competing interests}

The authors declare that they have no competing interests.

\section{Author details}

'Beijing Key Laboratory of Environment Friendly Management on Fruit Diseases and Pests in North China, Institute of Plant and Environment Protection, Beijing Academy of Agriculture and Forestry Sciences, Beijing 100097, China. ${ }^{2}$ Department of Plant Pathology, China Agricultural University, Beijing 100193, China. ${ }^{3}$ Dipartimento di Scienze e Tecnologie Agrarie, Alimentari, Ambientali e Forestali (DAGRI) - Sez. Patologia vegetale ed 
Entomologia, Università di Firenze, P. le delle Cascine 28, 50144 Firenze, Italy. ${ }^{4}$ Center of Excellence in Fungal Research, Mae Fah Luang University, Chiang Rai 57100, Thailand.

\section{Received: 9 November 2020 Accepted: 30 April 2021}

Published online: 25 May 2021

\section{References}

Abreo E, Martinez S, Bettucci L, Lupo S. Phaeomoniella chlamydospora and Phaeoacremonium spp. in grapevines from Uruguay. Phytopathol Mediterr. 2011:50:577-85

Akgul DS, Savas NG, Teker T, Keykubat B, Mayorquin JS, Eskalen A. Fungal trunk pathogens of sultana seedless vineyards in Aegean region of Turkey. Phytopathol Mediterr. 2015;54:380-93.

Baranek M, Armengol J, Holleinova V, Pecenka J, Calzarano F, Penazova E, et al. Incidence of symptoms and fungal pathogens associated with grapevine trunk diseases in Czech vineyards: first example from a north-eastern European grape-growing region. Phytopathol Mediterr. 2018;57:449-58.

Berraf-Tebbal A, Bouznad Z, Santos JM, Coelho MA, Peros JP, Phillips AJL. Phaeoacremonium species associated with Eutypa dieback and Esca of grapevines in Algeria. Phytopathol Mediterr. 2011;50:586-97.

Carbone I, Kohn LM. A method for designing primer sets for speciation studies in filamentous ascomycetes. Mycologia. 1999;91:553-6.

Chen H, Cui B-K. Multi-locus phylogeny and morphology reveal five new species of Fomitiporia (Hymenochaetaceae) from China. Mycol Prog. 2017;16:687701.

Chen W-P. Grape industry development report of Ningxia province in 2018. In: China agricultural research system for grape industry; 2018. p. 349.

Chu F-J, Chen W-Z. Grape industry development report of Hebei Province in 2018. In: Agricultural research system for grape industry; 2018. p. 137.

Cloete M. The characterization of the Basidiomycetes associated with esca disease in south African grapevines. Stellenbosch: PhD thesis, Stellenbosch University; 2015. https://doi.org/10.13140/RG.2.2.21115.62242.

Cloete M, Fischer M, Mostert L, Halleen F. A novel Fomitiporia species associated with esca on grapevine in South Africa. Mycol Prog. 2014;13:303-11.

Correia KC, Saraiva Camara MP, Guimaraes Barbosa MA, Sales R Jr, Agusti-Brisach C, Gramaje D, et al. Fungal trunk pathogens associated with table grape decline in northeastern Brazil. Phytopathol Mediterr. 2013;52:380-7.

Crous PW, Gams W, Wingfield MJ, van Wyk PS. Phaeoacremonium gen nov associated with wilt and decline diseases of woody hosts and human infections. Mycologia. 1996;88:786-96.

da Silva MA, Correia KC, Guimaraes Barbosa MA, Saraiva Camara MP, Gramaje D, Michereff SJ. Characterization of Phaeoacremonium isolates associated with petri disease of table grape in northeastern Brazil, with description of Phaeoacremonium nordesticola sp. nov. Eur J Plant Pathol. 2017;149:695-709.

Dai Y-C, Cui B-K, Decock C. A new species of Fomitiporia (Hymenochaetaceae, Basidiomycota) from China based on morphological and molecular characters. Mycol Res. 2008:112:375-80

Dissanayake AJ, Liu M, Zhang W, Chen Z, Udayanga D, Chukeatirote E, et al. Morphological and molecular characterisation of Diaporthe species associated with grapevine trunk disease in China. Fungal Biol. 2015a;119:28394.

Dissanayake AJ, Zhang W, Li X, Zhou Y, Chethana T, Chukeatirote E, et al. First report of Neofusicoccum mangiferae associated with grapevine dieback in China. Phytopathol Mediterr. 2015b;54:414-9.

Dissanayake AJ, Zhang W, Mei L, Chukeatirote E, Yan J, Li X, et al. Lasiodiplodia pseudotheobromae causes pedicel and peduncle discolouration of grapes in China. Australas Plant Dis Notes. 2015c;10:21.

Edwards J, Pascoe IG. Occurrence of Phaeomoniella chlamydospora and Phaeoacremonium aleophilum associated with petri disease and esca in Australian grapevines. Australas Plant Pathol. 2004;33:273-9.

Fischer M. A new wood-decaying basidiomycete species associated with Esca of grapevine: Fomitiporia mediterranea (Hymenochaetales). Mycol Prog. 2002;1: 315-24.

Fischer M. Biodiversity and geographic distribution of basidiomycetes causing Esca-associated white rot in grapevine: a worldwide perspective. Phytopathol Mediterr. 2006;45:S30-42.

Gatica M, Cesari C, Magnin S, Dupont J. Phaeoacremonium species and Phaeomoniella chlamydospora in vines showing "hoja de malvon" and young vine decline symptoms in Argentina. Phytopathol Mediterr. 2001:40:5317S24.
Gimenez-Jaime A, Aroca A, Raposo R, Garcia-Jimenez J, Armengol J. Occurrence of fungal pathogens associated with grapevine nurseries and the decline of young vines in Spain. J Plant Pathol. 2006;154:598-602.

Glass NL, Donaldson GC. Development of primer sets designed for use with the PCR to amplify conserved genes from filamentous Ascomycetes. Appl Environ Microbiol. 1995;61:1323-30.

Gramaje D, Mostert L, Groenewald JZ, Crous PW. Phaeoacremonium: from esca disease to phaeohyphomycosis. Fungal Biol. 2015;119:759-83.

Gramaje D, Urbez-Torres JR, Sosnowski MR. Managing grapevine trunk diseases with respect to etiology and epidemiology: current strategies and future prospects. Plant Dis. 2018;102:12-39.

Groenewald M, Kang JC, Crous PW, Gams W. ITS and beta-tubulin phylogeny of Phaeoacremonium and Phaeomoniella species. Mycol Res. 2001;105:651-7.

Gubler WD, Mugnai L, Surico G. In: Wilcox WF, Gubler WD, Uyemoto JK, editors. Compendium of grape diseases, disorders, and pests. 2nd ed. St Paul: APS Press; 2015.

Guerin-Dubrana L, Fontaine F, Mugnai L. Grapevine trunk disease in European and Mediterranean vineyards: occurrence, distribution and associated disease-affecting cultural factors. Phytopathol Mediterr. 2019;58:49-71.

Guo LD, Hyde KD, Liew ECY. Identification of endophytic fungi from Livistona chinensis based on morphology and rDNA sequences. New Phytol. 2000;147: 617-30.

Hall TA. BioEdit: a user-friendly biological sequence alignment editor and analysis program for windows 95/98/NT. Nucleic Acids Symp Ser (Oxf). 1999;41:95-8.

Hofstetter V, Buyck B, Croll D, Viret O, Couloux A, Gindro K. What if esca disease of grapevine were not a fungal disease? Fungal Divers. 2012;54:51-67.

Katoh K, Rozewicki J, Yamada KD. MAFFT online service: multiple sequence alignment, interactive sequence choice and visualization. Brief Bioinform. 2019;20:1160-6.

Kishino $\mathrm{H}$, Hasegawa M. Evaluation of the maximum likelihood estimate of the evolutionary tree topologies from DNA sequence data, and the branching order in Hominoidea. J Mol Evol. 1989:29:170-9.

Kuraku S, Zmasek CM, Nishimura O, Katoh K. aLeaves facilitates on-demand exploration of metazoan gene family trees on MAFFT sequence alignment server with enhanced interactivity. Nucleic Acids Res. 2013;41:W22-W8.

Larignon P, Dubos B. Fungi associated with Esca disease in grapevine. Eur J Plant Pathol. 1997;103:147-57.

Li X, Yan J, Kong F, Qiao G, Zhang Z, Wang Z. Botryosphaeria dothidea causing canker of grapevine newly reported in China. Plant Pathol. 2010;59:1170.

Luque J, Martos S, Aroca A, Raposo R, Garcia-Figueres F. Symptoms and fungi associated with declining mature grapevine plants in Northeast Spain. J Plant Pathol. 2009;91:381-90

Manawasinghe IS, Dissanayake AJ, Li X, Liu M, Wanasinghe DN, Xu J, et al. High genetic diversity and species complexity of Diaporthe associated with grapevine dieback in China. Front Microbiol. 2019:10:1936.

Miller MA, Pfeiffer W, Schwartz T. Creating the CIPRES Science Gateway for inference of large phylogenetic trees. 2010 Gateway Computing Environments Workshop (GCE). 2010. p. 1-8. https://doi.org/10.1109/GCE.2010.5676129.

Mohammadi H, Banihashemi Z, Gramaje D, Armengol J. Fungal pathogens associated with grapevine trunk diseases in Iran. J Agric Sci Technol. 2013;15: 137-50.

Mondello V, Songy A, Battison E, Pinto C, Coppin C, Trozel-Aziz P, et al. Grapevine trunk diseases: a review of fifteen years of trials for their control with chemicals and biocontrol agents. Plant Dis. 2018;102:1189-217.

Mostert L, Groenewald JZ, Summerbell RC, Gams W, Crous PW. Taxonomy and pathology of Togninia (Diaporthales) and its Phaeoacremonium anamorphs. Stud Mycol. 2006;54:1-113.

Mugnai L, Graniti A, Surico G. Esca (black measles) and brown wood-streaking: two old and elusive diseases of grapevines. Plant Dis. 1999;83:404-18.

O'Donnell K, Cigelnik E. Two divergent intragenomic rDNA ITS2 types within a monophyletic lineage of the fungus Fusarium are nonorthologous. Mol Phylogenet Evol. 1997;7:103-16.

OIV. 2019 statistical report on world Vitiviniculture: International Organization of vine and wine Intergovernmental Organization (OIV); 2019. http://www.oiv. int/en/technical-standards-and-documents/statistical-analysis/annual-a ssessment. Accessed 7 July 2020

Rambaut A. FigTree v1.4.4: tree figure drawing tool. 2018. https://github.com/ra mbaut/figtree/releases. Accessed 12 July 2020.

Romanazzi G, Murolo S, Pizzichini L, Nardi S. Esca in young and mature vineyards, and molecular diagnosis of the associated fungi. Eur J Plant Pathol. 2009;125: 277-90. 
Scheck HJ, Vasquez SJ, Gubler WD, Fogle D. First report of three Phaeoacremonium spp. causing young grapevine decline in California. Plant Dis. 1998;82:590.

Silvestro D, Michalak I. raxmIGUI: a graphical front-end for RAxML. Org Divers Evol. 2012;12:335-7.

Spies CFJ, Moyo P, Halleen F, Mostert L. Phaeoacremonium species diversity on woody hosts in the Western Cape Province of South Africa. Persoonia. 2018; 40:26-62.

Stamatakis A. RAxML version 8: a tool for phylogenetic analysis and post-analysis of large phylogenies. Bioinformatics. 2014;30:1312-3.

Surico G. Towards a redefinition of the diseases within the Esca complex of grapevine. Phytopathol Mediterr. 2009;48:5-10.

Surico G, Marchi G, Braccini P, Mugnai L. Epidemiology of esca in some vineyards in Tuscany (Italy). Phytopathol Mediterr. 2000;39:190-205.

Surico G, Mugnai L, Marchi G. Older and more recent observations on Esca: a critical overview. Phytopathol Mediterr. 2006;45:S68-86.

Swofford DL. PAUP*. Phylogenetic analysis using parsimony (*and other methods). Version 4.0b10. Sunderland: Sinauer Associates; 2002.

Úrbez-Torres JR, Peduto F, Striegler RK, Urrea-Romero KE, Rupe JC, Cartwright RD, et al. Characterization of fungal pathogens associated with grapevine trunk diseases in Arkansas and Missouri. Fungal Divers. 2012;52:169-89.

Wang BW. Analysis of Huailai County grape industry SWOT. Henan Agricultural University. Zhengzhou: Master thesis; 2012. https://kns.cnki.net/kcms/detail/ detail.aspx?FileName $=1015515654$.nh\&DbName $=$ CMFD2015.

Wang J. Effects of soil properties on wine grape and wine quality in different producing areas in the eastern foot of Helan Mountain. Ningxia University. Master thesis. 2018. https://kns.cnki.net/kcms/detail/detail.aspx?dbcode= CMFD\&dbname $=$ CMFD201901\&filename $=1018313337$. nh\& $\&=$ fOfjUBI $\mathrm{kra}$ dShOMFCBIFCq\%25mmd2BB\%25mmd2BdGEmTRxXnOFmPiQk\%25mmd2 FOtyGYpfiygtBeeoF\%25mmd2FWxcNM. Accessed 1 Apr 2018.

White TJ, Bruns T, Lee S, Taylor J. Amplification and direct sequencing of fungal ribosomal RNA genes for phylogenetics. In: Innis MA, Gelfand DH, Sninsky JJ, White TJ, editors. PCR Protocols: a Guide to Methods and Applications. London: Academic Press; 1990. p. 315-22. https://doi.org/10.1016/B9780-12-372180-8.50042-1.

Yan JY, Xie Y, Zhang W, Wang Y, Liu JK, Hyde KD, et al. Species of Botryosphaeriaceae involved in grapevine dieback in China. Fungal Divers. 2013;61:221-36

Ye QT, Manawasinghe IS, Zhang W, Mugnai L, Hyde KD, Li XH, et al. First report of Phaeoacremonium minimum associated with grapevine trunk diseases in China. Plant Dis. 2020;104:1259.

Ready to submit your research? Choose BMC and benefit from:

- fast, convenient online submission

- thorough peer review by experienced researchers in your field

- rapid publication on acceptance

- support for research data, including large and complex data types

- gold Open Access which fosters wider collaboration and increased citations

- maximum visibility for your research: over $100 \mathrm{M}$ website views per year

At $\mathrm{BMC}$, research is always in progress.

Learn more biomedcentral.com/submissions 